DUKE-TH-94-73

\title{
Lyapunov Exponent and Plasmon Damping Rate in Nonabelian Gauge Theories
}

\author{
T. S. Biró \\ Theory Division, MTA KFKI, RMKI, Pf. 49, H-1525 Budapest 114, Hungary \\ C. Gong, and B. Müller \\ Department of Physics, Duke University, Box 90305, Durham, NC 27708-0305
}

(Revised version January 26, 1995)

\begin{abstract}
We explain why the maximal positive Lyapunov exponent of classical $\mathrm{SU}(N)$ gauge theory coincides with (twice) the damping rate of a plasmon at rest in the leading order of thermal gauge theory.
\end{abstract}

Typeset using REVTEX 


\section{INTRODUCTION}

Numerical studies of Hamiltonian $\mathrm{SU}(N)$ lattice gauge theory in $(3+1)$ dimensions have shown that the gauge fields exhibit chaotic behavior in the classical limit [1]. The numerical value of the largest positive Lyapunov exponent $\lambda_{0}$ has been obtained for $\mathrm{SU}(2)$ and $\mathrm{SU}(3)$ with the result [1, 2 ]

$$
\lambda_{0}=c_{N} g^{2} E_{p}
$$

where $E_{p}$ is the average energy per plaquette, $c_{2} \approx 0.17$ for $\mathrm{SU}(2)$, and $c_{3} \approx 0.10$ for $\mathrm{SU}(3)$. For the SU(2) gauge theory the complete spectrum of Lyapunov exponents was obtained on small lattices [3]. These calculations, which follow the evolution of a classical gauge field configuration in Minkowski space, also showed that the energy density distribution on the lattice rapidly approaches a thermal distribution [四]. This finding confirms the expectation of a finite growth rate of the coarse-grained entropy density of the gauge field, which follows from the observation that the sum over all positive Lyapunov exponents at fixed energy density grows like the volume [3]. Hence, at any given level of coarse-graining, the classical gauge field "self-thermalizes" on a time scale of the order of the inverse Lyapunov exponent.

In order to determine the value of the maximal Lyapunov exponent $\lambda_{0}$, the evolution

of the gauge field configurations must be followed over periods $t_{0} \gg \lambda_{0}^{-1}$. The Lyapunov exponent is therefore effectively obtained for gauge fields that are members of a thermal ensemble, and we can identify the average energy per plaquette $E_{p}$ in (1) with that of a thermalized lattice. At high temperature the gauge field is a collection of weakly coupled harmonic oscillators, hence the average energy per independent degree of freedom of the classical gauge field is equal to the temperature $T$, yielding $E_{p}=\frac{2}{3}\left(N^{2}-1\right) T$ for $\operatorname{SU}(N)$. The factor $\frac{2}{3}$ accounts for the restrictions imposed by Gauss' law. We can therefore rewrite the result (1) as

$$
\lambda_{0}=\frac{2}{3} c_{N}\left(N^{2}-1\right) g^{2} T \approx \begin{cases}0.34 g^{2} T & (N=2), \\ 0.53 g^{2} T & (N=3) .\end{cases}
$$


As already noted in [4] these values for $\lambda_{0}$ coincide, apart from a factor 2, with those of the damping rate of a thermal plasmon at rest, obtained by Braaten and Pisarski [5] in the framework of thermal perturbation theory:

$$
\gamma_{0} \approx 6.635 \frac{N}{24 \pi} g^{2} T= \begin{cases}0.176 g^{2} T & (N=2), \\ 0.264 g^{2} T & (N=3) .\end{cases}
$$

The goal of the present article is to establish this connection and to explain the origin of the factor $\lambda_{0} / \gamma_{0}=2$.

We approach this goal in several steps. First we review the numerical "measurement" of the Lyapunov exponent in classical lattice gauge theory. We point out that the exponential growth rate of a small perturbation in the magnetic energy density used in those calculations is equal to twice of the growth rate of fluctuations in the elementary field variable, in the continuum limit the vector potential. This explains the factor 2 between $\lambda_{0}$ and $\gamma_{0}$.

In the next step we demonstrate that in classical calculations the linear perturbation propagation corresponding to the equations of motion of a chaotic dynamical system has in general a Fourier spectrum of imaginary frequencies. The Lyapunov exponent is equal to the magnitude of those imaginary frequencies.

Then we argue that the chaotic dynamics of the classical system acts like a thermal ensemble averaging the perturbation propagation equation over stochastic frequencies. The square of these frequencies can either be positive or negative. In this case the damping rate and the plasma frequency of the classical elementary field fluctuations are related to the mean value and the width of the probability distribution of frequency squares.

The final result of these considerations is that the Lyapunov exponent as defined in [1] measures twice the damping rate of classical gauge field fluctuations on the lattice. It is left to show that the quantum field theoretical calculation of the thermal damping rate at rest in hot perturbation theory in the leading $\mathcal{O}\left(g^{2} T\right)$ order survives in the classical $(\hbar \rightarrow 0)$ limit. We begin with the discussion of this point in order to establish connection with thermal quantum field theory. 


\section{COLLECTIVE PLASMA MODES}

We begin by briefly reviewing the derivation of the plasmon damping rate. Nonabelian gauge field fluctuations in a thermal background have been studied extensively in the framework of perturbation theory [6-10]. The gauge field develops massive collective modes (plasmons) with frequency $\omega(k)>k$ due to interaction with "hard" thermal gauge bosons, i.e.

excitations with energy of order $T$. The energy of a plasmon at rest is $m_{g} \equiv \omega(0)=\frac{1}{3} \sqrt{N} g T$ in $\mathrm{SU}(N)$ gauge theory. For our purpose it is important that the dispersion relation $\omega(k)$ can be obtained in the framework of semiclassical transport theory, where classical field fluctuations $a_{\mu}$ are coupled to the quantized thermal excitations of the gauge field [1]. The gauge invariant description of the collective modes requires the introduction of effective $n$-point vertices [8], which can be systematically derived from the effective action [12,13]:

$$
\mathcal{L}_{\mathrm{HTL}}\left(a_{\mu}\right)=-\frac{3}{2} m_{g}^{2} \int d \hat{n} \operatorname{tr}\left(f^{\mu \alpha} \frac{n_{\alpha} n_{\beta}}{(n \cdot D)^{2}} f_{\mu}^{\beta}\right)
$$

where $n_{\alpha}=(1, \hat{n})$ is a null four-vector, and the integral is over all directions of the spatial unit vector $\hat{n}$. $D^{\nu}$ stands for the gauge-covariant derivative. We have denoted the collective gauge potential $a_{\mu}$ and field strength $f_{\mu \nu}$ by lower-case letters to indicate that these describe fluctuations around a thermal background. Note that $\mathcal{L}_{\mathrm{HTL}}$ is a classical construction, with the sole exception that the plasmon rest mass $m_{g}$ depends on the energy distribution $n(\omega)=\left(e^{\hbar \omega / T}-1\right)^{-1}$ of quantized thermal excitations of the gauge field:

$$
m_{g}^{2}=\frac{2}{3} N g^{2} \frac{\hbar^{2}}{T} \int \frac{d^{3} k}{(2 \pi)^{3}} n(\omega)(1+n(\omega))=\frac{N}{9} \frac{g^{2}}{\hbar} T^{2} .
$$

At leading order in $g$, (5) is evaluated for hard thermal quanta with $\omega=|\vec{k}|$.

Braaten and Pisarski [5] showed that the collective plasmon modes are unstable due to the effective interaction (画. The plasmon damping rate $\gamma(k)$ is defined as imaginary part of the plasmon pole in the Feynman propagator corresponding to decaying plane wave solutions. The rate of instability for a plasmon at rest can be expressed as the imaginary part of the polarization function of the gauge field at the plasmon pole [14: 


$$
\gamma_{0} \equiv \gamma(0)=\frac{1}{2 m_{g}} \operatorname{Im}^{*} \Pi_{\mathrm{t}}\left(m_{g}+i 0,0\right),
$$

where the transverse polarization function ${ }^{*} \Pi_{\mathrm{t}}(\omega, \vec{k})$ only depends on soft modes described by (4). The plasmon rest mass exactly cancels from the expression (6) and the result (3) is a pure number multiplied by $g^{2} T$, which is a classical inverse length scale. In fact, the calculation explicitly makes use of the classical limit of the Bose distribution, $n(\omega) \rightarrow T / \hbar \omega$, in the evaluation of the loop integral (see eq. (23) of ref. [5]).

Since the effective action (4) can be derived from classical considerations [15], assuming a given spectrum of thermal excitations, it also applies to the collective excitations of the classical gauge field on a lattice. The sole modification is that the spectrum of thermal fluctuations is now given by the limit of the Bose distribution. Denoting the lattice spacing by $a$ we find

$$
m_{g}^{2} \rightarrow \frac{2}{3} N g^{2} T \sum_{\vec{k}} \frac{1}{\omega^{2}}=\frac{1}{3 \pi} N g^{2} \frac{T}{a}
$$

in the weak-coupling, large volume limit. The plasmon mass (7) is a purely classical quantity of dimension (length) $)^{-2}$ not containing $\hbar$, but it diverges in the continuum limit $a \rightarrow 0$. This is not surprising, since the lattice spacing serves as a cut-off that is required to regularize the ultraviolet divergences of the classical thermal gauge theory. The exponential growth rate of small classical field fluctuations is not affected by this divergence because it does not depend on the value of $m_{g}$, as mentioned above. The result (3) for the plasmon damping rate $\gamma_{0}$ remains valid if the correct plasmon mass $m_{g}$ in the effective action (4) is replaced by the value (7) for the classical gauge field defined on a lattice.

More intuitively, the independence of $\gamma_{0}$ from the value of $m_{g}$ can be understood as follows. The cross section for scattering of a thermal gluon on a slow plasmon is:

$$
\sigma \approx \frac{N^{2}}{N^{2}-1} \frac{g^{4} \hbar^{2}}{4 \pi \mu_{\mathrm{D}}^{2}}
$$

where $\mu_{\mathrm{D}}=\sqrt{3} m_{g}$ is the inverse Debye color screening length. The scattering rate $\nu$ is obtained by multiplying with the gluon density in the initial state and with the Bose factor in the final state, yielding: 


$$
\begin{aligned}
\nu & =2\left(N^{2}-1\right) \int \frac{d^{3} k}{(2 \pi)^{3}} n(\omega)(1+n(\omega)) \sigma \\
& =\frac{N^{2}-1}{N} \frac{T \mu_{\mathrm{D}}^{2} \sigma}{g^{2} \hbar^{2}} \approx \frac{N}{4 \pi} g^{2} T \approx \gamma_{0},
\end{aligned}
$$

where we have made use of (4). From this result, which has the same structure as the expression (3) for $\gamma_{0}$, it is obvious that the plasmon mass $m_{g}$ as well as $\hbar$ cancel from the scattering rate.

\section{LYAPUNOV EXPONENTS}

The Lyapunov exponents measure the growth rate of infinitesimal perturbations around an exact solution of the classical lattice Yang-Mills equations. Since the maximal Lyapunov exponent $\lambda_{0}$ was shown to be independent of the lattice spacing, we assume that we can work in the continuum limit whenever adequate. If $A_{\mu}(x, t)$ is an exact solution of the Yang-Mills equations, the linearized equation for a small perturbation $a_{\mu}(x, t)$ around $A_{\mu}$ is

$$
D^{2} a_{\mu}-D_{\mu} D_{\nu} a^{\nu}-2 i\left[F_{\mu \nu}, a^{\nu}\right]=0
$$

Here $D_{\mu}(A)=\partial_{\mu}-i\left[A_{\mu}, \quad\right]$ is the gauge covariant derivative where the bracket denotes the Lie algebra commutator, and $F_{\mu \nu}$ is the field strength tensor associated with the background field $A_{\mu}$.

The numerical approach to the determination of $\lambda_{0}$ proceeds by solving (10) for an arbitrary initial condition $a_{\mu}(x, 0)$ and measuring the growth rate of the norm of $a_{\mu}(x, t)$. To be precise, the maximal Lyapunov exponent was determined in [1,2] from the logarithmic growth rate of the "distance" between neighboring field configurations, defined on the lattice as

$$
\mathcal{D}\left[U_{\ell}^{\prime}, U_{\ell}\right]=\frac{1}{2 N_{p}} \sum_{p}\left|\operatorname{tr} U_{p}-\operatorname{tr} U_{p}^{\prime}\right|,
$$

where $U_{\ell}$ are the group valued link variables, $U_{p}$ denotes the elementary plaquette operator, and $N_{p}$ is the total number of spatial plaquettes. In the continuum limit, the distance measure (11) takes the form 


$$
\mathcal{D}\left[A_{\mu}^{\prime}, A_{\mu}\right] \propto \int d^{3} x\left|\operatorname{tr} B^{\prime}(x)^{2}-\operatorname{tr} B(x)^{2}\right|,
$$

where $B\left(B^{\prime}\right)$ are the magnetic fields associated with the gauge potential $A_{\mu}\left(A_{\mu}^{\prime}\right)$. In going from (111) to (12) we have suppressed the constant factor $\left(g^{2} a / 2 N_{p}\right)$, since we are interested

only in the growth rate of $(\ln \mathcal{D})$. For an infinitesimal perturbation $a_{\mu}$ that is a solution of the linearized equation (10), we obtain:

$$
\begin{array}{r}
\mathcal{D}\left[a_{\mu} \mid A_{\mu}\right] \equiv \mathcal{D}\left[A_{\mu}+a_{\mu}, A_{\mu}\right] \propto \\
\int d^{3} x\left|\operatorname{tr}\left(\frac{\partial\left(\operatorname{tr} B^{2}\right)}{\partial A_{\mu}} a_{\mu}\right)+\frac{1}{2} \operatorname{tr}\left(\frac{\partial^{2}\left(\operatorname{tr} B^{2}\right)}{\partial A_{\mu} \partial A_{\nu}} a_{\mu} a_{\nu}\right)\right| .
\end{array}
$$

The maximal Lyapunov exponent is then defined as

$$
\lambda_{0}\left[A_{\mu}\right]=\lim _{t_{0} \rightarrow \infty} \frac{1}{t_{0}} \ln \frac{\mathcal{D}\left[a_{\mu}\left(t_{0}\right) \mid A_{\mu}\right]}{\mathcal{D}\left[a_{\mu}(0) \mid A_{\mu}\right]} .
$$

In practice, every randomly chosen initial configuration $A_{\mu}(0)$ with a fixed average energy density has been found to yield the same value for the maximal Lyapunov exponent $\lambda_{0}$. The numerical calculations show that the maximal Lyapunov exponent depends only weakly on the lattice size and extrapolates smoothly to the limit of spatially homogeneous gauge potentials on a $1^{3}$ lattice. We take this as an indication that $\lambda_{0}$ is associated with long wavelength perturbations $a_{\mu}(x, t)$ in an appropriately chosen gauge.

\section{ERGODIC LIMIT}

We now propose to make use of the fact, noted in the Introduction, that the background gauge field $A_{\mu}(x, t)$ rapidly approaches thermal configurations, by replacing the long-time average of the growth rate of $(\ln \mathcal{D})$ by the canonical average over background gauge fields $A_{\mu}$, where the temperature $T$ is chosen such that the thermal energy density equals the average energy density of the time-dependent background field $A_{\mu}(x, t)$. The replacement of the temporal average by the canonical average relies on two conditions: The autocorrelation function of the background field $A_{\mu}(x, t)$ must decay on a time scale that is short 
compared with the time $t_{0}$ required for the calculation of the Lyapunov exponent, and the time evolution of the background field must be ergodic on the time scale $t_{0}$.

The ergodicity of the background gauge field is assured by its dynamical chaoticity on time scales long compared to the inverse of the positive Lyapunov exponents, hence the second condition is fulfilled [16]. On the other hand, if the first condition were violated, the Lyapunov exponent would depend on the starting configuration $A_{\mu}(x, t)$. In numerical studies [1-4] we have found that this is not the case. A direct study of the autocorrelation function performed by us has shown that the first condition is also satisfied. These conditions are in accordance with the $g^{2} T \ll g T \ll T$ hierarchy assumed in hot perturbative gauge theory.

The maximal Lyapunov exponent is then obtained from the relation

$$
\lambda_{0} \approx \frac{d}{d t} \ln \left\langle\mathcal{D}\left[a_{\mu}(t)\right]\right\rangle_{T}
$$

where the distance measure (13) in a thermal background is

$$
\begin{aligned}
\left\langle\mathcal{D}\left[a_{\mu}\right]\right\rangle_{T} & \propto \int d^{3} x \mid \operatorname{tr}\left(\left\langle\frac{\partial\left(\operatorname{tr} B^{2}\right)}{\partial A_{\mu}}\right\rangle_{T} a_{\mu}^{(T)}\right) \\
& +\frac{1}{2} \operatorname{tr}\left(\left\langle\frac{\partial^{2}\left(\operatorname{tr} B^{2}\right)}{\partial A_{\mu} \partial A_{\nu}}\right\rangle_{T} a_{\mu}^{(T)} a_{\nu}^{(T)}\right) \mid .
\end{aligned}
$$

The first term in (16) vanishes, because the thermal average of any quantity transforming under the adjoint representation is zero. In the second term, the thermal average projects on to the singlet part of $\partial^{2}\left(\operatorname{tr} B^{2}\right) / \partial A_{\mu} \partial A_{\nu}$, yielding

$$
\left\langle\mathcal{D}\left[a_{\mu}\right]\right\rangle_{T} \propto \int d^{3} x\left|\left\langle\frac{\partial^{2}\left(\operatorname{tr} B^{2}\right)}{\partial A_{\mu} \partial A_{\nu}}\right\rangle_{T} \operatorname{tr}\left(a_{\mu}^{(T)} a_{\nu}^{(T)}\right)\right| .
$$

Since the averaged value of $\mathcal{D}$ is quadratic in the field fluctuations $a_{\mu}^{(T)}$ the Lyapunov exponent defined through the magnetic energy distance measure is twice as large as the one defined by the dominant exponential growth rate of the fluctuations of the elementary field

$$
\lambda_{0}\left[A_{\mu}\right]=2 \lim _{t_{0} \rightarrow \infty} \frac{1}{t_{0}} \ln \frac{\left\|a_{\mu}\left(t_{0}\right)\right\|}{\left\|a_{\mu}(0)\right\|} .
$$




\section{CLASSICAL SPECTRAL FUNCTION}

Solving the classical equations of motion one deals with a problem essentially different from perturbative field theory: instead of investigating transition amplitudes between scattering states we follow the evolution of a given initial configuration from a time $t=0$ forwards. The appropriate method to analyze this evolution is not the Fourier transformation as in quantum field theory, but the Laplace transformation. Its inverse transformation is then calculated along a path which has all poles of the spectral function on its same side; the path's position is shifted accordingly, compared with the Fourier transformation.

The classical solution of the equations of motion for field perturbations therefore explores in forward time direction all poles of a free oscillator (or wave) equation. In case of chaotic Hamiltonian dynamics the solutions are both exponentially growing and damped giving rise to poles of the Laplace transform with positive as well as negative real parts.

Making the formal connection between Laplace and Fourier transformation through a complex rotation of the frequency variable, $s=i \omega$, the inverse Laplace transformation path runs above all poles in the complex $\omega$-plane. As a consequence in either case (oscillatory or chaotic) the integration path for the inverse Laplace transformation includes all poles for positive time and none for negative time while the Fourier transformation includes upper half plane poles for the advanced (negative time) and lower half plane poles for the retarded (positive time) propagator. 


\section{FIGURES}



FIG. 1. The integration paths in the complex frequency plane for the inverse Laplace $(L)$ and Fourier $(F)$ transformations.

The position of the poles obtained in a classical time — forward calculation may have in general both positive and negative imaginary parts. Therefore a better quantity for comparison is the spectral function which also considers poles in the whole complex $\omega-$ plane.

Summarizing this argument the position of all poles of a spectral function can be obtained from the linearized classical equations of motion for field perturbations (in the leading order of an $\hbar$ expansion), but the retarded and advanced propagators used to solve scattering problems in perturbative field theory discard the unsuitable ones due to their very definition. A positive Lyapunov exponent in Hamiltonian (energy conserving) dynamical systems, on the other hand, always occurs together with its negative counterpart — Liouville's theorem ensures it. Therefore studying positive exponential rates gives an information about the position of the poles of damped retarded and advanced propagators as well.

The growth or damping rate, or the oscillation frequency of small amplitude fluctuations in a classical dynamical system is studied by linearizing the classical equations of motion. This procedure leads to a new differential operator whose spectrum gives the poles of the classical spectral function. Odd parity under time reflection, real valuedness and normalization conditions then determine the relative weights of the pole terms.

The differential operator belonging to the linear perturbation propagation equation (10) 
is identical with the second variation of the classical action, $S^{\prime \prime}[A]$, taken at the background field configuration $A$ which is a solution of the classical equation of motion $S^{\prime}[A]=0$. Here the prime means variation with respect to $A$. Considering the generating functional of connected Green functions, the two point function is just the inverse of this differential operator,

$$
G\left[A, A^{\prime}\right]=\left\langle A A^{\prime}\right\rangle-\langle A\rangle\left\langle A^{\prime}\right\rangle=\left(S^{\prime \prime}[A]\right)^{-1},
$$

in the Gaussian approximation to the small amplitude fluctuations. So the linear perturbation propagation in classical equations of motion gives information about the saddle point approximated generating functional.

Now aiming at the description of long wavelength plasmon damping we may neglect spatial derivatives and write the general form of the classical, linearized perturbation propagation equation (10) schematically as

$$
\left[\frac{d^{2}}{d t^{2}}+\Omega^{2}(t)\right] a(t)=0 .
$$

The spectrum of this operator contains two poles on the real axis $\omega= \pm \Omega$ if $\Omega^{2}(t)$ is a positive constant. This case, familiar from zero-temperature perturbative field theory, describes small oscillations determining the real poles of the spectral function and the familiar retarded and advanced propagators. In classically chaotic, highly excited systems, however, it happens that $\Omega^{2}(t)$ is negative. This causes exponentially growing fluctuations - a typical source of chaotic behavior.

In order to gain a qualitative understanding about the (classical) spectral function of chaotic systems we consider $\Omega^{2}(t)$ as a Gauss-distributed stochastic variable [17. It can have both negative and positive values, and its time variation is replaced by the ensemble variation due to the ergodic property of classically chaotic dynamical systems discussed in the previous section. In this limit the probability distribution of the frequency squares, $P\left(\Omega^{2}\right)$, is determined by its two lowest moments,

$$
\left\langle\Omega^{2}\right\rangle=\alpha^{2}-\gamma^{2}
$$




$$
\left\langle\Omega^{4}\right\rangle-\left\langle\Omega^{2}\right\rangle^{2}=4 \alpha^{2} \gamma^{2}
$$

parametrized by two real parameters $\alpha$ and $\gamma$. This parametrization reflects the fact that while $\left\langle\Omega^{2}\right\rangle$ can either be positive or negative, the width of its distribution is always positive.

The stochastic average of the differential operator for the fluctuations has to be carried out on the quadratic level, because with the Gaussian distribution we assumed white noise property of the stochastic quantity. We get

$$
\begin{array}{r}
\left\langle\left(\omega^{2}-\Omega^{2}\right)^{2}\right\rangle=\omega^{4}-2\left\langle\Omega^{2}\right\rangle \omega^{2}+\left\langle\Omega^{4}\right\rangle= \\
(\omega-\alpha-i \gamma)(\omega-\alpha+i \gamma)(\omega+\alpha-i \gamma)(\omega+\alpha+i \gamma) .
\end{array}
$$

This result exhibits the symmetric four pole structure typical for a spectral function describing classical plasma oscillations

$$
\begin{array}{r}
\mathcal{A}(\omega)=\frac{1}{4 i \pi \omega} \quad \\
\quad\left(\frac{1}{\omega-\alpha-i \gamma}-\frac{1}{\omega-\alpha+i \gamma}\right. \\
\left.+\frac{1}{\omega+\alpha+i \gamma}-\frac{1}{\omega+\alpha-i \gamma}\right)
\end{array}
$$

yielding the Lorentz shape

$$
\mathcal{A}(\omega)=\frac{1}{\pi} \frac{2 \gamma \omega}{\left(\omega^{2}-\alpha^{2}-\gamma^{2}\right)^{2}+4 \gamma^{2} \omega^{2}}
$$

The relative signs of the pole terms follow from the definition of the spectral function as the difference between the advanced and retarded propagators and from its odd time parity $\mathcal{A}(-\omega)=-\mathcal{A}(\omega)$. The normalization factor $1 / 2 \omega$ ensures that

$$
\int_{-\infty}^{\infty} d \omega \omega \mathcal{A}(\omega)=1
$$

so in each mode exactly one boson is counted by the spectral function $\mathcal{A}(\omega)$ [18].

This particular, four pole spectral function describes a general solution of the stochastically averaged perturbation propagation equation which behaves like

$$
a(t)=A e^{i \alpha t-\gamma t}+B e^{-i \alpha t-\gamma t}+C e^{i \alpha t+\gamma t}+D e^{-i \alpha t+\gamma t} .
$$


After some initial oscillations the exponential growth dominates the long time behavior of $|a(t)|$. It is exactly this, which has been seen in numerical calculations. The conclusion of this argument is that the Lyapunov exponent of elementary field fluctuations averaged ergodically is equal to the classical gluon damping rate as expressed by the imaginary part of the pole positions in the spectral function $\mathcal{A}(\omega)$.

We note that in a recent publication [19] a similar Gaussian model for the chaotic instability in general Hamiltonian flows has been investigated. Our result presented above recovers the more general one of ref [19 for vanishing expectation value of the noisy oscillator frequency square $(\alpha=\gamma)$ after substituting a characteristic timescale $\tau=1 / \gamma$ in the general formula (19) of ref. [19].

\section{CLASSICAL AND QUANTUM GLUON DAMPING}

Finally we argue again that the leading order gluon damping rate (3) obtained in hot perturbative QCD (pQCD) is classical, i.e. it retains its value in the classical limit $\hbar \rightarrow 0$. This fact has been argued before in section II. Here we briefly reconstruct the argument and resolve some technical issues. This concludes our reasoning about the equality of the Lyapunov exponent of chaotic classical lattice gauge theory and the gluon damping rate at rest in a hot plasma.

The gluon damping rate in hot pQCD is obtained from the definition (6) dividing the imaginary part of the self energy by the thermal gluon mass $m_{g}=g T / \sqrt{\hbar}$. The general one-loop form of the self energy contains an integral over hard momenta, a factor of $g^{2}$ and the phase space distribution of thermal gluons

$$
\operatorname{Im} \Pi\left(m_{g}, 0\right)=g^{2} \hbar \int d^{4} k \frac{1}{k^{2}} n(k) f\left(k / m_{g}\right),
$$

where the complicated algebraic expression $f\left(k / m_{g}\right)$ depends only on scaled momentum variables. Using now the long wavelength approximation the phase space distribution of thermal gluons is replaced with its classical counterpart, $n(k) \approx T / \hbar \omega$, leading to 


$$
\operatorname{Im} \Pi\left(m_{g}, 0\right) \propto g^{2} T \int d \omega f\left(\omega / m_{g}\right)
$$

Scaling the integration variable with the Debye mass — which is of quantum origin containing the Planck constant — we see that the imaginary part of the 1-loop gluon self energy in a hot plasma is proportional to $m_{g}$. It follows that the gluon damping rate obtained using "classical" thermal gluons does not depend on the Debye mass and Planck's constant,

$$
\gamma=g^{2} T \int d x f(x)
$$

showing that the result (3) is essentially classical.

Finally, it is still to show whether non-pole contributions to the self energy in the field theoretical calculation do not interfere with the above arguments. The one-loop spectral function used there as an input contains a pole term picking up the zeroes $\omega(k)$ of the inverse propagator corresponding to collective plasma modes to the lowest order and a cut term describing the effect of scattering on thermally excited spacelike modes:

$$
\mathcal{A}(k, \omega)=Z(k) \delta\left(\omega^{2}-\omega(k)^{2}\right)+\beta(k, \omega) \Theta\left(k^{2}-\omega^{2}\right) .
$$

The cut coefficient $\beta(k, \omega)$ is related to the real and imaginary parts of the self energy $\Pi(k, \omega):$

$$
\beta(k, \omega)=\frac{\frac{1}{\pi} \operatorname{Im} \Pi}{\left(k^{2}-\omega^{2}+\operatorname{Re} \Pi\right)^{2}+(\operatorname{Im} \Pi)^{2}} .
$$

The respective self energies for the transverse and longitudinal excitations to leading order in hot perturbative QCD are 20]

$$
\Pi_{\mathrm{t}}(k, \omega)=m^{2} x^{2}\left(1+\frac{1-x^{2}}{2 x} \ln \frac{1+x}{1-x}\right)+\frac{i \pi}{2} m^{2} x\left(1-x^{2}\right)
$$

and

$$
\Pi_{\ell}(k, \omega)=k^{2}+m^{2}\left(2-x \ln \frac{1+x}{1-x}\right)-i \pi m^{2} x
$$

with $x=\omega / k$ and $m^{2}=3 m_{g}^{2} / 2$. Using these forms one obtains the following cut parts of the retarded Fourier transform of the spectral function, $\Delta(t, k)$, for small $k / m$ 


$$
\begin{array}{r}
\Delta_{\text {cut }, \mathrm{t}}(k, t) \rightarrow-2 \Theta(t) \frac{k}{m^{2}} \\
\int_{0}^{1} d x \frac{4 x\left(1-x^{2}\right) \sin k t x}{4 x^{4}\left(1+\frac{1-x^{2}}{2 x} \ln \frac{1+x}{1-x}\right)^{2}+x^{2}\left(1-x^{2}\right)^{2}}
\end{array}
$$

and

$$
\Delta_{\text {cut }, \ell}(k, t) \rightarrow-2 \Theta(t) \frac{k}{2 m^{2}} \int_{0}^{1} d x \frac{x \sin k t x}{\left(2-x \ln \frac{1+x}{1-x}\right)^{2}+x^{2}} .
$$

Since the integrand is bounded, the cut contribution cannot grow exponentially with time and hence does not contribute to the maximal Lyapunov exponent (15). In fact, the cut contribution vanishes in the long wavelength limit $k \rightarrow 0$.

This leaves us with the pole part, which remains finite in this limit.

\section{SUMMARY}

This concludes our argument establishing a connection between the classical Lyapunov exponent and the gluon damping rate in hot perturbative QCD. We note that some elements of the argument are heuristic, in particular, the replacement of the long-time average of the growth rate of fluctuations around a specific field configuration by the thermal average. This reasoning assumes that the growth rate, or equivalently the plasmon damping rate, depends only on coarse-grained properties of the gauge field. We believe that this is so, because the one-loop calculation of the damping rate $\gamma_{0}$ only involves soft loop momenta [8] and hence does not depend on details of the short-distance fluctuations of the gauge field.

Because of the general nature of our argument, we conjecture that the complete spectrum of Lyapunov exponents obtained in [3] reflects the spectrum of damping rates $\gamma(k)$ of excitations in a thermal bath. If this were true, it would confirm our assumption that $\gamma(k) \leq \gamma_{0}$. Since, at present, it is not known whether $\gamma(k)$ is a quantity with a classical limit for $k \neq 0$, the identification with the Lyapunov spectrum remains a conjecture. We finally note that if the correspondence between ergodic and canonical averages holds up for other physical quantities, transport coefficients of nonabelian gauge fields at the classical 
scale $\left(g^{2} T\right)$, such as magnetic screening [21] or color diffusion [22], could possibly also be calculated by real-time evolution of classical gauge fields on a lattice.

Acknowledgements: We thank U. Heinz, S. G. Matinyan, H. B. Nielsen, G. K. Savvidy, and M. Thoma for illuminating discussions and the referee for pointing out a mistake in our original manuscript. This work was supported in part by the U.S. Department of Energy (grant DE-FG05-90ER40592) and in part by the Collaboration Agreement between the Norwegian Research Council (NFR) and the Hungarian Academy of Science (MTA) (grant 422.92/001). One of us (B.M.) thanks the Physics Department of Tokyo Metropolitan University, especially H. Minakata, for their hospitality and support during his visit there. T.S.B. acknowledges the hospitality and the support of Physics Department of Bergen University and of L. P. Csernai during his visit there. 


\section{REFERENCES}

[1] B. Müller and A. Trayanov, Phys. Rev. Lett. 68, 3387 (1992).

[2] C. Gong, Phys. Lett. B298, 257 (1993).

[3] C. Gong, Phys. Rev. D49, 2642 (1994).

[4] T. S. Biró, C. Gong, B. Müller, and A. Trayanov, Int. J. Mod. Phys. C5, 113 (1994).

[5] E. Braaten and R. D. Pisarski, Phys. Rev. D42, 2156 (1990).

[6] V. V. Klimov, Zh. Eksp. Teor. Fiz. 82, 336 (1982) [Sov. Phys. JETP 55, 199 (1982)].

[7] H. A. Weldon, Phys. Rev. D26, 1394 (1982).

[8] E. Braaten and R. D. Pisarski, Nucl. Phys. B337, 569 (1990); Phys. Rev. Lett. 64, 1338 (1990).

[9] J. Frenkel and J. C. Taylor, Nucl. Phys. B334, 199 (1990).

[10] J. P. Blaizot and E. Iancu, Nucl. Phys. B390, 589 (1993); Phys. Rev. Lett. 70, 3376 (1993); Nucl. Phys. B417, 608 (1994).

[11] U. Heinz, Ann. Phys. (NY) 161, 48 (1985); ibid 168, 148 (1986).

[12] J. C. Taylor and S. Wong, Nucl. Phys. B346, 115 (1990).

[13] R. Efraty and V. P. Nair, Phys. Rev. Lett. 68, 2891 (1992); Phys. Rev. D47, 5601 (1993).

[14] The sign conventions in ref. 5 are such that a positive imaginary part of $* \Pi$ implies a negative imaginary part of the pole energy. In writing (6) we have already continued ${ }^{*} \Pi_{\mathrm{t}}$ to Minkowski space.

[15] P. F. Kelly, O. Liu, C. Lucchesi, and C. Manuel, Phys. Rev. Lett. 72, 3461 (1994).

[16] Note that the infrared limit of the Yang-Mills equation has been shown not to be 
completely ergodic, with some very small regions of stable trajectories remaining in phase space. See: P. Dahlquist and G. Russberg, Phys. Rev. Lett. 65, 2837 (1990); R. Marcinek, E. Pollak, and J. Zakrzewski, Phys. Lett. B327, 67 (1994). It is unknown, if such a phenomenon persists in the case of spatially dependent Yang-Mills fields.

[17] see e.g. N. G. van Kampen, Phys. Rep. 24, 171 (1976).

[18] G. Baym and N. D. Mermin, J. Math. Phys. 2, 232 (1961).

[19] L. Casetti, R. Livi and M. Pettini, Phys. Rev. Lett. 74, 375 (1995).

[20] R. D. Pisarski, Physica A158, 146 (1989).

[21] A. D. Linde, Phys. Lett. B96, 389 (1980); T. S. Biró and B. Müller, Nucl. Phys. A561, 477 (1993).

[22] A. V. Selikhov and M. Gyulassy, Phys. Lett. B316, 373 (1993); H. Heiselberg, Phys. Rev. Lett. 72, 3013 (1994). 